\title{
Calidad de vida y variables psicológicas que afectan la adherencia al tratamiento anti-retroviral en pacientes mexicanos con infección por VIH/SIDA
}

\author{
Itzel Gutiérrez-Gabriel', Jesús Godoy-Guinto², Herzain Lucas-Alvarado³, Benito Pineda-Germán², \\ Eduardo Vázquez-Cruz ${ }^{4}$ Maricarmen Hernández-De la Rosa ${ }^{2}$ y Francisca Sosa-Jurado ${ }^{5}$
}

\section{Quality of life and psychological variables affecting adherence to antiretroviral treatment in Mexican patients with HIV/AIDS}

Background: Antiretroviral treatment (ART) is essential in HIV/AIDS patients. Suppressing viral load requires strict adherence to ART in addition to the patient's commitment to treatment. The failure of ART is mainly due to lack of adherence, which may in turn be due to poor quality of life and/or to psychological variables. Aim: To determine the quality of life and psychological variables and adherence to ART, in patients with HIV/AIDS. Material and Method: 160 patients diagnosed with HIV/AIDS and with ART were included. The MOS SF-36 and VPAD-24 instruments, a socio-demographic survey, and clinical data were collected. Quantitative and qualitative associations were made between the variables. Results: The adherence to ART was associated with avoidance of depressive behavior and with the absence of addictions. Depressive behavior associated with addictions. $87 \%$ of patients ranked in the best quality of life. Below the average of the general health score were males, with MSM sexual orientation, single, in vitality at $\geq 38$ years, in corporal pain and with social function to three ART schemes. Conclusion: Good adherence to ART was associated with avoiding depressive behavior and with non-addictions and not associated with quality of life.

Keywords: HIV; AIDS; adherence to antiretrovirals; quality of life.

Palabras clave: VIH; SIDA; adherencia a anti-retrovirales; calidad de vida.

\section{Introducción}

E n Latinoamérica y el Caribe, casi 2.100 .000 personas tienen la infección por VIH/SIDA; los países caribeños de habla no hispana tienen las incidencias más altas de esta enfermedad en los últimos cinco años ${ }^{1}$. Durante este mismo período, países como Chile y Cuba han reportado un incremento gradual de la incidencia de VIH/SIDA ${ }^{1,2}$.

El comportamiento de la epidemia de infección por VIH/SIDA en México en los últimos 10 años (2005-2017) ha permanecido estable ${ }^{3}$. De los casos acumulados de 1983 a 2015, la vía de transmisión ha sido las relaciones sexuales no protegidas en $95,2 \%{ }^{4}$, Otra vía de transmisión es el uso de drogas inyectables; en años recientes, una situación que se torna emergente en la frontera norte de México ${ }^{5}$. La prevalencia en adultos de 20 a 49 años en 2007 fue de $0,37 \%^{6}$ y en $>15$ años en 2014 de $0,2 \%{ }^{4}$. La tasa de mortalidad a causa del SIDA ha disminuido heterogéneamente debido a que la tendencia de la mortalidad varía por condición de situación laboral, género, edad y por la región de residencia ${ }^{7}$. La proporción entre hombres y mujeres con infección por VIH/SIDA ha permanecido $4: 1^{3}$ y los grupos de población más afectados en México son los hombres que tiene sexo con otros hombres (HSH) y personas transgénero ${ }^{1}$.

En 1997 fue introducido el tratamiento anti-retroviral (TAR) en México para los beneficiarios de la seguridad social estatal y paraestatal, y en 2003 para trabajadores del sector informal, estrategia que permitió notablemente disminuir la mortalidad en 32\% desde 1997 a $2011^{8}$. En los últimos años se ha visto que el TAR ha ayudado a mejorar la calidad de vida de los pacientes con infección por VIH/SIDA 9 .

El TAR es imprescindible para los pacientes con esta condición debido a que disminuye la carga viral, pero se requiere de una estricta adherencia al mismo. La buena adherencia produce descenso de la replicación viral, lo que se asocia con mantener un sistema inmune funcional ${ }^{10}$. En un estudio en América Latina se obtuvo una adherencia al TAR de alrededor de $70 \%$, porcentaje comparable a la de otras poblaciones en regiones en desarrollo, aunque puede ser inferior a los niveles requeridos para una supresión de la replicación viral exitosa a largo plazo ${ }^{11}$.
Jefatura de Medicina Familiar, Unidad de Medicina Familiar $\mathrm{N}^{\circ}$ 6, Instituto Mexicano del Seguro Social, Puebla, Puebla, México. ${ }^{2}$ Servicio de Atención Familiar, Unidad de Medicina Familiar No 57, Instituto Mexicano del Seguro Social, Puebla, Puebla, México. ${ }^{3}$ Servicio de Medicina Interna, Hospital General de Zona No 20 Instituto Mexicano del Seguro Social, Puebla, Puebla, México. 4Jefatura de Educación e Investigación, Unidad de Medicina Familiar $\mathrm{N}^{\circ} 6$, Instituto Mexicano del Seguro Social, Puebla, Puebla, México. ${ }^{5}$ Centro de Investigación Biomédica de Oriente, Instituto Mexicano del Seguro Social, Metepec, Atlixco, Puebla, México.

Los autores declaran no tener conflictos de interés. Fuente de financiamiento: Presupuesto asignado a la Unidad de Medicina Familiar No 57 (UMF-57), Instituto Mexicano del Seguro Social, Puebla, Puebla, México.

Recibido: 10 de enero de 2018 Aceptado: 22 de enero de 2019

Correspondencia a: Francisca Sosa Jurado sosajurado@hotmail.com 
Con estos datos se demuestra la importancia de determinar la falta de adherencia al TAR en los pacientes con infección por VIH/SIDA. Primero es necesario detectar qué factores desalientan a los pacientes para tener buena adherencia, para implementar programas que los reduzcan dependiendo de las características de la población estudiada.

Como segundo punto, se debe evitar la aparición de cepas con resistencia anti-retroviral en pacientes sin previo tratamiento ${ }^{12}$, ya que es el riesgo que conlleva la no adherencia, lo que la convierte en un problema de Salud Pública.

La adherencia al TAR en los pacientes con esta dolencia no implica solamente una toma constante de un fármaco; es una variable dependiente que está en función de varios factores que pueden o no afectarla, e incluso puede ser difícil detectar con claridad qué variables están contribuyendo. Así, pueden ser los factores sociodemográficos como edad, género, estrato social, nivel educativo, la situación laboral, la orientación sexual, las relaciones familiares, la relación con el personal de salud, la nutrición, los hábitos personales, el grupo con infección por VIH/SIDA estudiado ${ }^{13-15}$, factores cognitivos como la desinformación sobre la enfermedad, respecto al TAR las bajas expectativas a su efectividad ${ }^{16}$, los efectos secundarios causados, la dificultad de seguir el tratamiento, incluida la carga posológica ${ }^{17}$, y otros factores como el impacto emocional relacionado con el diagnóstico y la convivencia con una enfermedad como VIH/SIDA que puede causar depresión, principalmente en los pacientes jóvenes recién diagnosticados. Éstos tienden a recurrir al consumo de alcohol y sustancias psicoactivas, lo que conduce a corto plazo a la aparición de trastornos psiquiátricos a temprana edad y que repercuten más en la no adherencia al TAR ${ }^{15,18}$. La depresión se ha asociado con daño al sistema inmunológico de los pacientes con infección por VIH y, en consecuencia, los conduce más rápidamente a la etapa más grave de la enfermedad ${ }^{19}$. Por ello, se debe prestar atención en detectar pacientes con comportamientos que conducen a la depresión, para así ayudar a estas personas a mantener fortalecido su sistema inmunológico.

La calidad de vida de los pacientes con infección por VIH actualmente está poco afectada, en gran parte debido al TAR; esto se ha reportado en España ${ }^{9}$, Colombia ${ }^{20,21}$, Brasil $^{21} \mathrm{y}$ en pacientes mexicanos con infección por VIH que viven cerca de la frontera E.U.A.-México ${ }^{22}$. Aunque, no así en mujeres indígenas con la infección, donde el estigma en sus comunidades, la depresión, y su no resiliencia a la enfermedad se asocian en forma negativa con la calidad de vida ${ }^{23}$.

La asociación entre la adherencia al TAR y una mejor calidad de vida no ha sido estudiada en México. VenturaCerda no observó correlación entre la adherencia al TAR y la calidad de vida9 . Por otro lado, un estudio reportó que los pacientes clasificados como no adherentes al TAR obtuvieron los peores puntajes en casi todos los dominios de calidad de vida ${ }^{24}$

\section{Objetivos}

- Determinar adherencia al TAR y variables psicológicas.

- Determinar calidad de vida en ocho dimensiones.

- Determinar la asociación entre adherencia al TAR con variables demográficas, clínicas, psicológicas y de calidad de vida.

- Determinar la asociación entre las ocho dimensiones de calidad de vida con adherencia al TAR, variables demográficas, clínicas, y psicológicas en pacientes con diagnóstico de VIH/SIDA que asisten a la Unidad de Medicina Familiar No 57 (UMF 57) del Instituto Mexicano del Seguro Social (IMSS), en la Ciudad de Puebla, Puebla, México.

\section{Material y Método}

\section{Tipo de estudio}

Se realizó un estudio comparativo, analítico, transversal, entre enero de 2017 y julio de 2017, que incluyó 160 pacientes adscritos a la UMF 57, IMSS, Puebla, Puebla, México.

\section{Población y muestra}

Fueron 160 pacientes con diagnóstico de infección por VIH/SIDA.

\section{Criterios de inclusión}

Mayores de 18 años, género femenino y masculino, con TAR, que aceptaron participar y contestar las evaluaciones y la encuesta.

\section{Criterios de exclusión}

Pacientes cursando con enfermedad oportunista o con algún trastorno psiquiátrico.

\section{Aspectos éticos}

Se tuvieron presentes los principios de la Declaración de Helsinki. El estudio se apegó a la Ley General de Salud en materia de investigación (Secretaria de Salud, México). Les fue dada a los participantes una carta de consentimiento informado, donde se le informó el propósito del estudio, así como los riesgos y beneficios, asegurando la protección de sus datos y con la garantía de que si querían retirarse del estudio esto no afectaría los servicios que les otorga ser derechohabiente IMSS. El proyecto fue aprobado por el Comité Local de Investigación y Ética en Investigación en Salud 2104, de la Unidad de Medicina Familiar No.6, Puebla, Puebla, México. Número de registro (R-2016-2104-29). 


\section{Método de recolección de datos}

El médico tratante invitó a participar en el estudio durante la consulta médica a los pacientes que cumplieron con los criterios de inclusión y exclusión. El médico encargado de la investigación les indicó que se requería de 20 a 30 min para contestar una encuesta socio-demográfica y las evaluaciones MOS SF-16 y VPAD-24.

\section{Datos demográficos y clínicos}

Se obtuvieron de la encuesta socio-demográfica y los datos clínicos como carga viral (CV), cuenta de linfocitos $\mathrm{CD} 4+/ \mathrm{mm}^{3}$, el esquema de tratamiento, y los años de diagnóstico fueron tomados del expediente clínico de los pacientes.

\section{Evaluaciones}

Calidad de vida con el Medical OutcomeStudy Short Form (MOS SF-36).

Les fue aplicado el instrumento MOS SF- $36^{20}$, que es aplicable a personas sanas o con alguna enfermedad para evaluar programas de salud y detectar los beneficios a la salud de un tratamiento. Está compuesto por 36 ítems que valoran los estados tanto positivos como negativos de la salud y cubre ocho dimensiones: Función física (FF), desempeño físico (DF), dolor corporal (DC), salud general (SG), vitalidad (V), función social (FS), desempeño emocional (DE) y salud mental (SM). Utiliza una escala que tiene un recorrido que va desde 0 a 50 (que se cataloga como el peor estado de salud para esa dimensión), y de 51 hasta 100 (catalogado como el mejor estado de salud). Se determinó la confiabilidad interna para este instrumento mediante el $\alpha$ de Cronbach $\geq 0,7$.

\section{Adherencia al TAR y variables psicológicas con el instrumento VPAD-24}

Se evaluó la adherencia al TAR y otras variables psicológicas utilizando el instrumento VPAD-2425, el que contiene 30 ítems y se basa en un modelo psicológico de salud biológica y, en consecuencia, permite delimitar con precisión la dimensión psicológica que es pertinente a la salud en general y al problema de la adhesión al tratamiento, en particular en personas seropositivas frente al VIH. Se utilizaron los ítems para evaluar: el comportamiento que tiende a evitar depresión (27, 28, 29 y 30), las adicciones a alcohol o drogas $(4,17,26)$, el conocimiento del paciente sobre la enfermedad $(19,20,21$ y 22), y la adherencia al TAR ( 6 al 18), con una escala que recorre de 1 (nunca) a 5 (siempre). Se determinó la confiabilidad interna mediante el $\alpha$-Cronbach's $\geq 0,700$.

\section{Análisis de datos}

Para el cálculo del tamaño de muestra se usó la fórmula de Freeman ${ }^{26}$. Se calcularon frecuencias, porcentajes, y la media. Para las diferencias entre el puntaje de cada una de las 8 dimensiones de calidad de vida, asociada a cada una de las variables demográficas, clínicas y psicológicas se usó prueba t de Student para muestras independientes. Para las asociaciones entre variables nominales se usó $\chi^{2}$ y la prueba exacta de Fisher y para el análisis multivariado la regresión logística multinominal. Todas las pruebas estadísticas se realizaron con el programa IBM $^{\circledR}$ SPSS $^{\circledR}$ Statistics Versión 22.

\section{Resultados}

\section{Encuesta socio-demográfica y datos clínicos}

El $85,6 \%$ de los pacientes fue de sexo masculino, el promedio de edad en general fue de 38,8 años, $51,2 \%>38$ años, $69 \%$ no casados, $33,1 \%$ con preparación básica, $53,8 \%$ con orientación sexual HSH, $60,6 \%$ con $\leq 7$ años de diagnóstico VIH/SIDA, 8,75\% con recuento de linfocitos CD $4+<200 / \mathrm{mm}^{3}$, y $26,5 \%$ con CV detectable (Tabla 1).

Tabla 1. Datos demográficos, clínicos, adherencia al TAR y variables psicológicas en pacientes con infección por VIH/SIDA que asisten a la UMF-57 en Puebla, México

n (\%)

$\begin{array}{lc}\text { Datos demográficos } & \\ \text { Género hombre } & 137(85,6) \\ \text { Promedio de edad (IC95\% ) } & 38,8(27,2-50,4) \\ \text { Pacientes > 38 años } & 82(51,2) \\ \text { Educación básica (primaria y secundaria) } & 58(33,1) \\ \text { Educación media o superior } & 102(63,7) \\ \text { Orientación sexual hombre/hombre (HSH) } & 86(53,8) \\ \text { Orientación heterosexual } & 74(46,2) \\ \text { Estado civil casado } & 37(23,1)\end{array}$

Datos clínicos

Años de diagnóstico de infección por VIH (promedio) $\quad 7,2(6,1-8,2)$

Pacientes $\leq 7$ años de diagnóstico

Pacientes con recuento CD4 $+<200 / \mathrm{mm}^{3}$

$97(60,6)$

Pacientes con carga viral $>40$ copias $/ \mathrm{ml}$

$14(8,75)$

$42(26,5)$

Esquemas TAR

$1 \quad 64(40,0)$

$233(20,6)$

$3 \quad 36(22,5)$

4,6

5,7 u 8

$11(6,9)$

$16(10,0)$

Adherencia a TAR y variables psicológicas

Adherencia a TAR

Conocimiento de la enfermedad

$104(65)$

Con tendencia a evitar comportamiento depresivo

Con adicciones (alcohol o drogas)

$101(63.1)$

$70(47.4)$

$49(30.6)$

Esquemas de TAR: 1 (atazanvir-tenofovir/emtricitabina-rinotavir), 2 (tenofovir/emtricitabinarinotavir/lopinavir), 3 (tenofovir/emtricitabina-efavirenz), 4 (nevirapina- tenofovir/emtricitabina), 5 (abacavir-lamividina-efavirez), 6 (raltegravir-darunavir-rinotavir), 7 (abacavir/3TC-nevirapina), 8 (abacavir -rinotavir- atazanavir-). 


\section{Variables de adherencia y psicológicas}

El $65 \%$ de los pacientes tuvo adherencia al TAR, $61,1 \%$ con conocimiento de la enfermedad, $90(56,3 \%)$ de los pacientes con tendencia a comportamiento depresivo, y en 30,6\% se detectó alguna adicción (alcohol o drogas) (Tabla 1).

\section{Calidad de vida en ocho dimensiones}

El análisis de la calidad de vida mostró que en las dimensiones FF, DF, FS, DS y SM, 97,2\% de los pacien- tes se ubicó en el puntaje de 51 a 100 (mejor calidad de vida). (Figura 1). En las dimensiones V y DC, 7,5\% de los pacientes se ubicó en el puntaje 1 a 50 (peor calidad de vida) y en la dimensión SG 2,5\%. La media del puntaje de cada una de las 8 dimensiones fue: la FF 91,8, DF 93,7, DC 87,1, SG 76,6, V 73,9, FS 88,7, DE 87,5 y SM 83,0 (Tabla 2). Frecuencia y porcentaje de pacientes con puntaje por debajo de la media: en la FS 52(32,5\%), DF $35(21,8 \%)$, DC 57(35,6\%), SG 78(48,8\%), V 75(46,8\%), FS 57(35,6\%), DE 57(35,6\%) y SM 77(48,1\%) (Figura 1).

Tabla 2. Asociación de las ocho dimensiones de calidad de vida con variables demográficas, clínicas, adherencia al TAR y variables psicológicas

\begin{tabular}{|c|c|c|c|c|c|c|c|c|}
\hline \multirow[t]{2}{*}{ Puntaje } & \multicolumn{8}{|c|}{ Ocho dimensiones de calidad de vida } \\
\hline & $\begin{array}{l}\text { Función } \\
\text { física }\end{array}$ & $\begin{array}{l}\text { Desempeño } \\
\text { físico }\end{array}$ & $\begin{array}{l}\text { Dolor } \\
\text { corporal }\end{array}$ & $\begin{array}{c}\text { Salud } \\
\text { general }\end{array}$ & Vitalidad & $\begin{array}{c}\text { Función } \\
\text { social }\end{array}$ & $\begin{array}{c}\text { Desempeño } \\
\text { emocional }\end{array}$ & $\begin{array}{l}\text { Salud } \\
\text { mental }\end{array}$ \\
\hline Media $\pm I C 95 \%$ & $91,8 \pm 1,7$ & $93,7 \pm 2,1$ & $87,1 \pm 2,9$ & $76,6 \pm 1,9$ & $73,9 \pm 2,4$ & $88,7 \pm 2,2$ & $87,5 \pm 2,9$ & $83,0 \pm 2,1$ \\
\hline
\end{tabular}

Variables

Demográficas

Género

\begin{tabular}{|c|c|c|c|c|c|c|c|c|}
\hline & & & & & & & & \\
\hline Masculino & 92,3 & 93,2 & 86,0 & $75,8^{*}$ & 74,5 & 87,9 & 87,3 & 83,2 \\
\hline Femenino & 88,9 & 97,3 & 93,5 & 81,6 & 71,1 & 93,4 & 89,2 & 81,9 \\
\hline & & & & & & & & \\
\hline$\geq 38$ & 90,9 & 93,4 & 84,9 & 76,9 & $70,0^{*}$ & 86,6 & 89,2 & 81,3 \\
\hline$<38$ & 92,7 & 94,0 & 89,3 & 76,7 & 77,4 & 90,6 & 85,4 & 84,6 \\
\hline
\end{tabular}

Orientación sexual

$\mathrm{HSH}$

Sí

$\begin{array}{lll}91,8 & 93,6 & 86,6\end{array}$

$93,4 \quad 86,3$

$73,4^{*}$
$86,3 \quad 79,5$

$\begin{array}{ll}73,4^{*} & 71,7 \\ 79,5 & 75,5\end{array}$

75,5

89,7

88,2

82,9

Estado civil

Casado
Sí

Sí

92,8

94,9

88,9

$80,6 \quad 74,0$

74,5

88,9

88,4

88,0

82,6

Características clínicas

Recuento de CD4 $+<200 \mathrm{~mm}^{3}$

Sí

No

$\begin{array}{ll}91,7 & 93,5 \\ 92,9 & 95,8 \\ 91,6 & 93,5 \\ 91,9 & 94,7 \\ & \\ 88,0 & 93,3 \\ 91,8 & 95,8 \\ 91,2 & 93,1 \\ 93,3 & 91,7 \\ 94,0 & 92,0\end{array}$

91,6

86,5

81,3

79,7

$73,2^{*}$

88,0

93,8

93,5

86,8

81,6

$\mathrm{CV}>40$ copias $/ \mathrm{ml}$

Sí

No

Esquemas TAR

Esquemas 5,7-8

Esquema 1

Esquema 2

Esquema 3

Esquema 4, 6

94,0

$\begin{array}{lll}85,4^{*} & 77,8 & 74,1\end{array}$

91,8

76,1

73,6

87,3

86,9

89,3

8

92,0

$80,1^{*} \quad 74,2 \quad 69,7$

$\begin{array}{lll}80,1 * & 74,2 & 69,7 \\ 88,0 & 77,2 & 74,3\end{array}$

$87,3 \quad 77,0 \quad 73,3$

87,0

75,0

87,0

78,9

76,0

71,3

$82,3^{*}$
88,2
86,0
92,2
91,9

$79,5^{\top}$

87,1

89,3

90,3

84,8

83,0

83,1

Variables psicológicas

Conocimiento de la enfermedad

Sí

No

91,2

93,3

92,7

95,6

86,4

88,3

75,7

78,3

75,5

88,1

87,9

82,3

Evitar comportamiento depresivo

Sí

No

92,6

94,3

87,6

90,8

93,3

86,7

78,0

75,5

76,4

$72,0^{\top}$

89,8
87,8

88,0

86,0

$77,2^{\top}$

83,7

84,7

82,4

89,3

${ }^{*} p<0,05$ (diferencia significativa) celdas gris oscuro, $p \geq 0.05 \leq 0.1$ ( ${ }^{\top}$ ) tendencia (no diferencia significativa) celda gris claro. Esquemas de TAR: 1 (atazanvir-tenofovir/ emtricitabina-rinotavir), 2 (tenofovir/emtricitabina-rinotavir/lopinavir), 3 (tenofovir/emtricitabina-efavirenz), 4 (nevirapina- tenofovir/emtricitabina), 5 (abacavir-lamividinaefavirez), 6 (raltegravir-darunavir-rinotavir), 7 (abacavir/3TC-nevirapina), 8 (abacavir -rinotavir- atazanavir). 
Asociación entre la adherencia al TAR con las variables demográficas, clínicas, de calidad de vida en ocho dimensiones y las psicológicas

Sólo dos variables independientes estuvieron asociadas con tener adherencia al TAR: la tendencia a evitar comportamiento depresivo $(\mathrm{RR}=4,10 ; \mathrm{p}=0,000)$ y la ausencia de adicciones $(R R=6,10 ; p=0,000)$ (Tabla 3$)$.

\section{Asociación entre tener tendencia a un tener comportamiento depresivo y las variables} demográficas, clínicas, y las variables psicológicas

Una variable fuertemente asociada a la tendencia a tener un comportamiento depresivo fue la presencia de adicciones $(\mathrm{RR}=3,2 ; \mathrm{p}=0,003)$, y mostró cierta tendencia, (diferencia no significativa), la variable esquemas de tratamiento 5,7 o $8(\mathrm{RR}=2,7 ; \mathrm{p}=0,05)$ (Tabla 4).

\section{Asociación de las ocho dimensiones de calidad de vida con variables demográficas, clínicas y la adherencia al TAR y variables psicológicas}

Si bien la gran mayoría de los pacientes estuvo en el puntaje de mejor calidad de vida, entre 32,5 y $48,1 \%$ de ellos se ubicaron por debajo del promedio del puntaje de alguna de las dimensiones de calidad de vida.

Por debajo del promedio de la dimensión DC (con diferencia significativa), se encontraron a pacientes caracterizados por tener $\mathrm{CV}>40$ copias $/ \mathrm{ml}$ y con alguno de los esquemas de tratamiento 5, 7 u 8 (Tabla 2). Por debajo del promedio de la dimensión SG (con diferencia significativa) se encontraron a pacientes con las siguientes

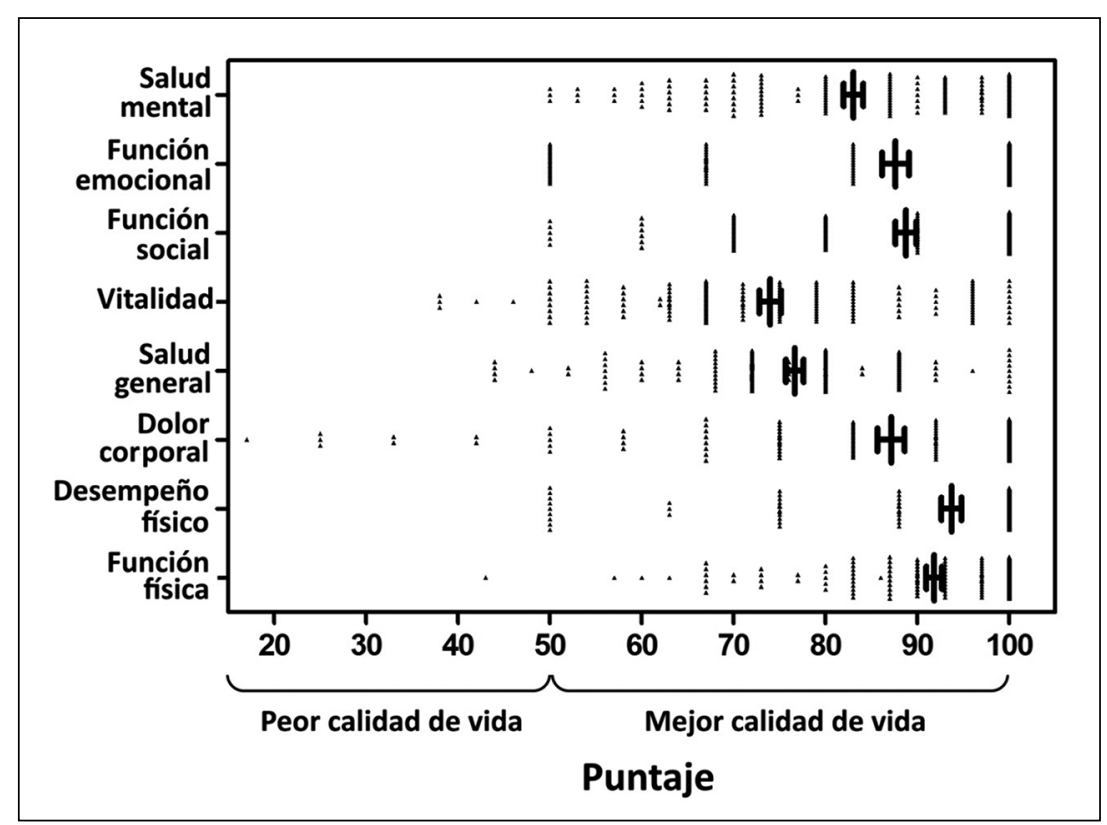

Figura 1. Calidad de vida en ocho dimensiones de los pacientes con infección por VIH/Sida que asisten a la UMF-57 en Puebla, México.

características: sexo masculino, con orientación sexual $\mathrm{HSH}$, no casados, con cuenta de CD4 $>200 / \mathrm{mm}^{3}$ (Tabla 2). Por debajo del promedio de la dimensión $\mathrm{V}$, (con diferencia significativa), se ubicaron pacientes que se caracterizaron por tener $\geq 38$ años, y con cuenta de CD4

\begin{tabular}{|c|c|c|c|c|c|c|}
\hline \multirow[b]{2}{*}{ Factor } & \multirow[b]{2}{*}{ Pacientes } & \multicolumn{2}{|c|}{ Análisis univariado } & \multirow[b]{2}{*}{ Valor $\mathrm{p}$} & \multicolumn{2}{|c|}{ Análisis multivariado } \\
\hline & & $\begin{array}{l}\text { Adherencia al tratamiento } \\
\qquad n \quad(\%)\end{array}$ & $\begin{array}{l}\text { Riesgo relativo } \\
\text { IC95\% }\end{array}$ & & $\begin{array}{l}\text { Riesgo relativo } \\
\text { IC95\% }\end{array}$ & Valor $p$ \\
\hline \multicolumn{7}{|c|}{ Estado civil casado } \\
\hline Sí & 37 & $19(51)$ & $1,3(0,98-1,85)$ & 0,038 & - & - \\
\hline No & 123 & $85(69)$ & & & & \\
\hline \multicolumn{7}{|c|}{ Conocimiento de la enfermedad } \\
\hline Sí & 101 & $71(70)$ & $1,8(0,95-3,63)$ & 0,048 & - & - \\
\hline No & 59 & $33(55,9)$ & & & & \\
\hline \multicolumn{7}{|c|}{ Tendencia a evitar un comportamiento depresivo } \\
\hline Sí & 70 & $58(82,0)$ & $1,6(1,29-2,0)$ & 0,000 & $4,10(1,8-9,3)$ & 0,000 \\
\hline No & 90 & $46(51,1)$ & & & & \\
\hline \multicolumn{7}{|c|}{ Tener adicciones (alcohol o drogas) } \\
\hline Sí & 49 & $17(34,6)$ & & & & \\
\hline No & 111 & $85(76,5)$ & $2,20(1,48-3,2)$ & 0,000 & $6,10 \quad(2,7-13,4)$ & 0,000 \\
\hline
\end{tabular}


Tabla 4. Análisis univariado y multivariado de variables que favorecenla tendencia a un comportamiento depresivo en pacientes con infección por VIH que asisten a la UMF-57 en Puebla, México

\begin{tabular}{|c|c|c|c|c|c|c|}
\hline \multirow[b]{2}{*}{ Factor } & \multicolumn{4}{|c|}{ Análisis univariado } & \multicolumn{2}{|c|}{ Análisis multivariado } \\
\hline & Pacientes & $\begin{array}{c}\text { Tendencia depresión } \\
\text { n (\%) }\end{array}$ & $\begin{array}{l}\text { Riesgo relativo } \\
\text { IC95\% }\end{array}$ & Valor $\mathrm{p}$ & $\begin{array}{l}\text { Riesgo relativo } \\
\text { IC95\% }\end{array}$ & Valor $p$ \\
\hline \multicolumn{7}{|c|}{ Conocimiento de la enfermedad } \\
\hline Sí & 101 & $45(44,5)$ & $1,49(1,2-1,9)$ & 0,003 & ----------- & ---------- \\
\hline No & 59 & $45(76,2)$ & & & & \\
\hline \multicolumn{7}{|c|}{ Adicciones } \\
\hline Sí & 49 & $37(75,5)$ & $3,3(1,5-7,1)$ & 0,001 & $3,2(1,4-6,9)$ & 0,003 \\
\hline No & 111 & $53(47,7)$ & & & & \\
\hline \multicolumn{7}{|c|}{ Esquema TAR 5, 7-8* } \\
\hline Sí & 16 & $13(81,0)$ & $2,7(0,99-10)$ & 0,05 & ------- & --------- \\
\hline No & 147 & $77(54,4)$ & & & & \\
\hline
\end{tabular}

$>200 / \mathrm{mm}^{3}$, el no conocer la enfermedad y el no evitar un comportamiento depresivo, (diferencia no significativa), asociado a estar por debajo de la media del puntaje de la V (Tabla 2). Por debajo del promedio de la dimensión FS, (con diferencia significativa), y por debajo del promedio de las dimensiones DE y SM, (no diferencia significativa), se encontraron pacientes caracterizados por estar con alguno de los esquemas de tratamiento 5, 7 u 8 (Tabla 2).

\section{Discusión}

Nuestros resultados muestran que el género y la edad promedio de los pacientes fueron similares a los datos reportados, donde la población más afectada en México es los pacientes $<34$ años ${ }^{3}$. Sesenta por ciento de nuestros pacientes tuvieron $<38$ años y edad promedio de 38 años, hallazgo similar a un estudio que incluyó a 11 países $^{21} \mathrm{y}$ por abajo del promedio reportado en España de 46 años ${ }^{9}$.

La adherencia al TAR obtenido en este estudio con VPDA-24 fue de 65\%. Recientemente, una revisión sistemática de adherencia al TAR reportó una adherencia de alrededor de $70 \%$ para América Latina con varios instrumentos para determinarla y donde fueron incluidos trabajos con pacientes mexicanos ${ }^{11}$.

La adherencia al TAR en este estudio estuvo asociada a las variables: tendencia a evitar comportamiento depresivo y ausencia de adicciones (Tabla 3). La depresión es común en un cierto porcentaje de pacientes con infección por VIH, ya que es propiciada por la misma infección ${ }^{18}$. Está bien documentado que la depresión y las adicciones están asociadas a la no adherencia al TAR ${ }^{19}$. Estudios dirigidos para detectar síntomas de depresión en pacientes con infección por VIH/SIDA en países latinoamericanos también coinciden en este punto ${ }^{27,28}$. Nosotros detectamos a $82,0 \%$ de los pacientes con tendencia a evitar comportamiento depresivo y a 76,5\% con ausencia de adicciones que tuvieron adherencia al TAR (Tabla 3). Aunque, tener conocimiento de la enfermedad también favorece la adherencia al TAR (Tabla 3 ).

El no tener conocimiento de la enfermedad estuvo asociado, en el análisis univariado, a tener comportamiento depresivo (Tabla 4), resultado que nos hace replantear el reforzamiento de este punto en nuestros pacientes. Tender a tener un comportamiento depresivo estuvo asociado a las adicciones (Tabla 4), formándose un círculo cerrado comportamiento depresivo-adicciones que puede estar afectando la adherencia al TAR en algunos de nuestros pacientes.

El riesgo de caer en depresión es mayor en personas jóvenes recién diagnosticadas con infección por VIH/SIDA, los que optan por el uso de sustancias psicoactivas por el impacto que les causa el diagnóstico ${ }^{19}$. En este estudio, las adicciones estuvieron presentes en 34,6 y $26,8 \%$ entre los menores y mayores de 38 años, respectivamente (dato no mostrado). Una limitante de nuestra investigación es el no haber empleado un instrumento para detectar síntomas específicos de depresión en pacientes con esta afección, pero en un futuro estudio multidisciplinario, se puede considerar identificarlos, como en los estudios efectuados en Chile $^{27}$ y Colombia ${ }^{28}$.

A la gran mayoría de nuestros pacientes se les encontró en el rango de puntaje de mejor calidad de vida (Figura 1). Resultados similares dentro de este rango se han obtenido 
en pacientes mexicanos con infección por VIH/SIDA viviendo en la frontera norte ${ }^{22}$, y en un estudio de metaanálisis que incluyó a 11 países, entre estos a Colombia y Brasil ${ }^{21}$. Aunque esto es distinto en pacientes con infección por VIH/SIDA de comunidades rurales marginadas, sobre todo en mujeres, donde principalmente los factores psicológicos afectan su calidad de vida ${ }^{23}$.

Entre 32,5 y $48,8 \%$ de nuestros pacientes estuvieron por debajo del promedio del puntaje en alguna de las dimensiones de calidad de vida (Figura 1). En la dimensión SG, 77 pacientes tuvieron un puntaje por debajo del promedio. Se caracterizaron por tener algunas o todas estas variables: masculinos, con orientación sexual HSH, no casados y cuenta CD4 $+>200 / \mathrm{mm}^{3}$ (Tabla 2). En el más reciente estudio de calidad de vida en pacientes mexicanos con infección por VIH/SIDA, se asociaron a una baja puntuación en salud física a las variables género, orientación sexual, estado civil y tiempo de diagnóstico, pero no establecieron claramente sus resultados, debido al tamaño de muestra y al sesgo en responder la pregunta sobre la orientación sexual ${ }^{29}$.

En la dimensión DC, los 57 pacientes encontrados en el puntaje por debajo del promedio se caracterizaron por tener alguna o las dos variables $\mathrm{CV}$ detectable y/o tener alguno de los esquemas de tratamiento 5, 7, u 8 (Tabla 2). Los 16 pacientes con alguno de los esquemas de tratamiento 5, 7, u 8 tuvieron un puntaje promedio de 82,3 en la dimensión FS marcando una diferencia significativa con respecto al promedio obtenido en esta dimensión con los pacientes con alguno de los esquemas de tratamiento 1 , 2, 3, 4, o 6 (Tabla 2). Los esquemas de tratamiento 5,7, $\mathrm{u}$ 8 tuvieron en común el fármaco abacavir, el que se ha reportado que puede causar reacciones neuro-psiquiátricas ${ }^{30}$. El esquema 5 contiene efavirenz, que tiene el riesgo de manifestaciones neuro-psiquiátricas en pacientes con antecedentes de abuso de sustancias ${ }^{31}$. Estos resultados deben ser tomados con precaución ya que se requiere de más investigación al respecto.

La adherencia al TAR en este estudio no estuvo asociada a alguna de las ocho dimensiones de calidad de vida, resultado similar a lo obtenido por Ventura-Cerda en España ${ }^{9}$, pero no así al estudio de De Oliviera e Silva en Brasil ${ }^{24}$. La adherencia al TAR no estuvo asociado a tener $\mathrm{CV}$ no detectable o recuento de linfocitos CD4+ $>200 / \mathrm{mm}^{3}$; quizá se deba a que la encuesta VPAD-24 cuestiona en el curso de la semana pasada para medir adherencia al TAR.

Nosotros detectamos 46 pacientes con adherencia al TAR entre los 90 con tendencia a comportamiento depresivo (Tabla 3). A la depresión se le ha asociado con daño al sistema inmunológico de los pacientes con infección por $\mathrm{VIH}^{10,19}$. En este estudio, el recuento de linfocitos CD4+ de los pacientes con un comportamiento depresivo estuvo por debajo del recuento de linfocitos CD4+ de los pacientes que evitaron tener un comportamiento depresivo (dato no mostrado).

El conjunto de resultados obtenidos en este estudio, nos indican lo complejo que puede ser esta enfermedad, así como establecer variables que favorezcan la adherencia o la no adherencia ${ }^{15}$. Y que su manejo y tratamiento debe ser personalizado ${ }^{8}$. En los pacientes con orientación sexual HSH, su salud general al parecer está más afectada con respecto a los demás pacientes de este grupo estudiado $\mathrm{y}$, si son mayores de 38 años, también está disminuida su vitalidad. Los datos emitidos de UNAIDS indican que este grupo de pacientes tiene la mayor prevalencia de infección por VIH/SIDA en México'.

En conclusión, la adherencia al TAR en nuestros pacientes con infección por VIH estuvo afectada por la tendencia a un comportamiento depresivo y a la presencia de adicciones; pero no estuvo asociada a las dimensiones de calidad de vida alguna. El 97,2\% de los pacientes estuvo en el puntaje de mejor calidad de vida. En las dimensiones SG, V y DC, por debajo del promedio se ubicó a las variables: masculinos, $\mathrm{HSH}$, no casados, con recuento CD4+>200/ $/ \mathrm{mm}^{3},>38$ años, con CV detectable y con alguno de los esquemas de tratamiento 5,7 u 8 .

Agradecimientos: A Gerardo Santos López y Paulina Cortés Hernández por la revisión del texto.

\section{Resumen}

Introducción: El tratamiento anti-retroviral (TAR) es indispensable en pacientes con infección por VIH/ SIDA; suprimir la carga viral requiere de un estricto apego a éste, por compromiso del paciente. El fracaso del TAR es primordialmente por falta de adherencia, que puede ser debida a una deficiente calidad de vida y/o a variables psicológicas. Objetivo: Determinar la calidad de vida, variables psicológicas y la adherencia al TAR, en pacientes con infección por VIH/SIDA. Material y Método: Se incluyeron 160 pacientes con diagnóstico de infección por VIH/SIDA y con TAR. Se recabaron los instrumentos MOS SF-36 y VPAD-24, una encuesta demográfica, y datos clínicos. Se hicieron asociaciones cuantitativas y cualitativas entre las variables. Resultados: La adherencia al TAR estuvo asociada con evitar comportamiento depresivo y con ausencia de adicciones. El comportamiento depresivo se encontró asociado con las adicciones. Un $87 \%$ de pacientes estaba en el rango de mejor calidad de vida. Por debajo del promedio del puntaje de salud general estuvieron masculinos, con orientación sexual HSH, solteros, en la vitalidad a los $\geq 38$ años, en dolor corporal y función social a tres esquemas TAR. Conclusión: La buena adherencia al TAR estuvo asociada a evitar comportamiento depresivo y a la ausencia de adicciones y no se asoció a la calidad de vida. 


\section{Referencias bibliográficas}

1.- UNAIDS DATA 2017. Joint United Nations Programme on HIV/SIDA (UNAIDS). Global and Regional data HIV/SIDA pag 12-16 y pág. 112-149.http://www.unaids.org/sites/default/ files/media_asset/2017_data-book_en.pdf

2.- Departamento de Epidemiología. División de Planificación Sanitaria. Ministerio de Salud de Chile. Estimaciones poblacionales sobre VIH en Chile 2017 SPECTRUM, ONUSIDA. Rev Chilena Infectol 2018; 35: 642-8.

3.- Vigilancia epidemiológica de casos de VIH/SIDA en México. Registro Nacional de casos de SIDA actualización al cierre de 2017; Secretaria de Salud, dirección general de epidemiología, Centro Nacional para la prevención y control de VIH/SIDA (CENSIDA).https://www.gob.mx/cms/uploads/ attachment/file/328393/RN_Cierre_2017.pdf

4.- Posadas-Robledo F J. La epidemia de VIH y SIDA en México. Hoja informativa 01; Secretaria de Salud, Dirección general de epidemiología, Centro Nacional para la prevención y control de HIV/SIDA (CENSIDA) 2015.http://www.censida.salud. gob.mx/descargas/principal/la_epidemia_ deVIHysidaMexico.pdf.

5.- Mehta S R, Chaillon A, Gaines T L, González-Zúñiga P E, Stockman J K, Almanza-Reyes $\mathrm{H}$, et al. Impact of public safety policies on human immunodeficiency virus transmission dynamics in Tijuana, Mexico. Clin Infect Dis 2018; 66:758-64. doi: 10.1093/cid/cix884.

6.- Valdespino J L, García-García M L, Conde-González C J, Olaiz-Fernández G, Palma O, Sepúlveda J. Prevalencia de infección por VIH en la población adulta en México: Una epidemia en ascenso y expansión. Salud pública Méx 2007; 49: S386-94. https://www.scielosp.org/article/ssm/content/ raw/?resource_ssm_path $=/$ media/assets/spm/ v49s3/09.pdf.

7.- Hernández-Ávila J E, Palacio-Mejía L S, Hernández-Romieu A, Bautista-Arredondo S, Sepúlveda-Amor J, Hernández-Ávila M. Implementation and operational research: effect of universal access to antiretroviral therapy on HIV/AIDS mortality in Mexico 1990-2011. J Acquir Immune Defic Syndr 2015 J; 69: e1008. doi: 10.1097/QAI.0000000000000645.

8.- Tratamiento antirretroviral del paciente adulto con infección por VIH. Ciudad de México. Instituto Mexicano del Seguro Social. 16/03/2017. Disponible en: http://imss.gob.mx/ profesionales-salud/gpc.

9.- Ventura- Cerdá J M, Martín-Conde M T, Morillo-Verdugo R, Tébenes-Cortés M, Casado-Gómez M A. Adherence, satisfaction and health-related quality of life in HIVinfected patients with antiretroviral therapy in Spain. The ARPAS study. FarmHosp 2014; 38: 291-9. doi: 10.7399/fh.2014.38.4.7404.

10.- Balbaryski J, Simonte K, Urteneche I, Candi M, Gaddi E, Barboni G. Antiretroviral treatment adherence and its association with TCD4+ lymphocyte subsets in children with HIV/ AIDS. Medicina (B Aires) 2013; 73: 324-30. PMID: 23924530.

11.- Costa-J D M, Torres T S, Esteves C L, Mendes L P. Adherence to antiretroviral therapy for HIV/AIDS in Latin America and the Caribbean: Systematic review and metaanalysis. J Int AIDS Soc 2018; 21: e25066. doi: 10.1002/jia2.25066.

12.- Ávila-Ríos S, García-Morales C, Matías-Florentino M, Romero-Mora K A, Tapia-Trejo D, Quiroz-Morales V S, et al. Pretreatment HIV-drug resistance in Mexico and its impact on the effectiveness of firstline antiretroviral therapy: a nationally representative 2015 WHO survey. Lancet HIV 2016; 3:e579-91. doi: 10.1016/S23523018(16)30119-9.

13.- Varela-Arevalo M T, Hoyos-Hernández P A. La adherencia al tratamiento para el VIH/SIDA: más allá de la toma de antirretrovirales. Rev Salud Pública 2015; 17:528-40.DOI: http://dx.doi.org/10.15446/ rsap.v17n4.38429.

14.- Pérez-Salgado D, Compean-Dardón M S, Staines-Orozco M G, Ortiz-Hernández L. Satisfaction with healthcare services and adherence to antiretroviral therapy among patients with hiv attending two public institutions. Rev Invest Clin 2015; 67:80-8. PMID: 25938840

15.- Boretzki J, Wolf E, Wiese C, Noe S, Balogh A, Meurer A, et al. Highly specific reasons for nonadherence to antiretroviral therapy: results from the German adherence study. Patient Prefer Adherence 2017; 11: 1897-906. doi: 10.2147/PPA.S141762.

16.- Varela-Arévalo M T, Salazar-Torres I C, Correa- Sánchez D. Adherencia al tratamiento en la infección por VIH/SIDA. Consideraciones teóricas y metodológicas para su abordaje. Acta Col de Psicología 2008; 11:101-13.

17.- Pérez-Rodríguez I, Pérez-Salgado D, Compeán-Dardón M S, Staines-Orozco M G, Ortiz-Hernández L. Efectos secundarios del tratamiento antirretroviral y apego en pacientes con VIH de dos instituciones públicas. MedIntMéx2016; 32:396-406.http://www. medigraphic.com/pdfs/medintmex/mim-2016/ mim164d.pdf.

18.- Bhatia R, Hartman C, Kallen M A, Graham J, Giordano T P. Persons newly diagnosed with
HIV infection are at high risk for depression and poor linkage to care: results from the Steps Study. AIDS Behav2011; 15: 1161-70. doi: 10.1007/s10461-010-9778-9.

19.- González J S, Batchelder A W, Psaros C, Safren S A. Depression and HIV/AIDS treatment non adherence: A review and meta-analysis. J Acquir Immune Defic Syndr 2011; 58:181-7. doi: 10.1097/ QAI.0b013e31822d490a.

20.- Cardona-Arias, Pelaez-Venegas L, López-Saldarriaga J, Duque-Molina M, Leal-Alvarez. Calidad de vida relacionada con la salud en adultos con VIH/SIDA, Medellín, Colombia. Biomédica 2011; 31: 532-44. https://www.revistabiomedica.org/index.php/ biomedica/article/view/422/787.

21.- Cardona-Arias J A, Higuita-Gutiérrez L F. Impact of HIV/AIDS on quality of life: meta-analysis 2002-2012.Rev Esp Salud Publica 2014; 88: 87-101. doi: 10.4321/S113557272014000100006 .

22.- Zúñiga M L, Blanco E, Jesse J, Brennan M A, Scolari R, Irina B A, et al. Binational careseeking behavior and health-related quality of life among HIV-infected Latinos in the U.S.-Mexico border region. J Assoc Nurses AIDS Care 2011; 22: 162-72. doi: 10.1016/j. jana.2010.09.001.

23.- Holtz C, Sowell R, Van Brackle L, Velásquez G, Hernández-Alonso V. A quantitative study of factors influencing quality of life in rural Mexican women diagnosed with HIV. J Assoc Nurses AIDS Care 2014; 25: 555-67. doi: 10.1016/j. jana.2014.03.002.

24.- De Oliviera e Silva A C, Reis R K, Almeida Noguera J, Gir E. Calidad de vida, características clínicas y adhesión al tratamiento de personas viviendo con VIH/SIDA. Rev Lat Am Enfermagem 2014; 22:994-1000. doi: 10.1590/01041169.3534.2508

25.- Piña-López J A, Corrales-Rascón A E, Mungaray-Padilla K, Valencia-Vidrio M A. Instrumento para medir variables psicológicas y comportamientos de adhesión al tratamiento en personas seropositivas frente a VIH (VPAD24). Rev Panam Salud Pública 2006; 19: 217 28. DOI: $10.1590 / \mathrm{S} 1020-49892006000400001$.

26.- Ortega-Calvo M, Cayuela-Domínguez A. Unconditioned logistic regression and simple size: a bibliographic review. Rev Esp Salud Pública 2002; 76: 85-93. PMID: 12025266.

27.- Varela M, Galdames S. Depresión y adhesión a terapia anti-retroviral en pacientes con infección por VIH atendidos en el Hospital San Pablo de Coquimbo, Chile. Rev Chilena Infectol 2014; 31:323-8.http://dx.doi. org/10.4067/S0716-10182014000300011. 
28.- Cardona-Duque D V, Medina-Pérez O, Herrera-Castaño S M, Orozco-Gómez P A. Depresión y factores asociados en personas viviendo con VIH/SIDA en Quindio, Colombia Rev Haban Cienc Med 2016; 15: 941-54.http:// scielo.sld.cu/pdf/rhcm/v15n6/rhcm10616.pdf.

29.- Quintero-Quiñónez J F, Goodman-Meza
D, Burgos-Regil J L, Muñoz-Carvajal F A, Zúñiga-de Nuncio M L, Ornelas-Aguirre J M. Quality of life in persons with human immunodeficiency virus infection in Mexico. Rev Med Inst Mex Seg Soc 2017; 56: 126-31. http://www.medigraphic.com/pdfs/imss/im2018/im182b.pdf.
30.- Foster R, Taylor C, Everall I P. More on abacavir-induced neuropsychiatric reactions. AIDS 2004; 18:2449.PMID: 15622330

31.- Cespedes M S, Aberg J A. Neuropsychiatric complications of antiretroviral therapy. Drug Saf 2006; 29: 865-74.DOI:10.2165/00002018200629100-00004. 